\title{
Yin Zhi Huang, a traditional Chinese herbal formula, ameliorates diet-induced obesity and hepatic steatosis by activating the AMPK/SREBP-1 and the AMPK/ACC/CPT1A pathways
}

\author{
Qunyan Yao ${ }^{1,2 \#}$, Shuyu $\mathrm{Li}^{1,2 \#}$, Xinlai Cheng ${ }^{3}$, Yanting Zou ${ }^{1,2}$, Yue Shen ${ }^{1,2}$, Shuncai Zhang ${ }^{1,2}$ \\ ${ }^{1}$ Department of Gastroenterology and Hepatology, Zhongshan Hospital, Fudan University, Shanghai 200030, China; ${ }^{2}$ Shanghai Institute of Liver \\ Diseases, Shanghai 200030, China; ${ }^{3}$ Institute of Pharmacy and Molecular Biotechnology, University of Heidelberg, Heidelberg, Germany \\ Contributions: (I) Conception and design: All authors; (II) Administrative support: Q Yao, S Li, S Zhang; (III) Provision of study materials or \\ patients: Q Yao; (IV) Collection and assembly of data: Q Yao, S Li, Y Zou, Y Shen; (V) Data analysis and interpretation: Q Yao, S Li, X Cheng; (VI) \\ Manuscript writing: All authors; (VII) Final approval of manuscript: All authors. \\ \#These authors contributed equally to this work. \\ Correspondence to: Shuncai Zhang. Department of Gastroenterology and Hepatology, Zhongshan Hospital, Fudan University, Shanghai 200030, \\ China. Email: zhang.shuncai@zs-hospital.sh.cn.
}

\begin{abstract}
Background: Yin Zhi Huang $(\mathrm{YZH})$ is a formula composed of Artemisia scoparia, Gardeniae fructus, Scutellaria baicalensis Georgi, and Lonicerae Japonicae Flos. Most of the components are eaten as food in Asia. Here, we evaluated the role of $\mathrm{YZH}$ on a high-fat diet (HFD)-induced obesity and hepatic steatosis.

Methods: Male C57BL/6J mice were fed with normal-chow diet, HFD, and HFD with low- or high-dose YZH for 16 weeks. Body weight gain, adipose mass, and plasma lipids levels were measured to evaluate the effect of YZH on obesity. Liver weight and staining methods on liver tissues were used to determine hepatic steatosis. The expression of involved genes and proteins were screened with qRT-PCR and immunoblotting, respectively.

Results: The results showed that $\mathrm{YZH}$ significantly reduced body weight gain, adipose mass, and the size of adipocytes, while did not affect food intake in HFD-fed mice. H\&E staining, bodipy staining, and oil red $\mathrm{O}$ staining displayed that $\mathrm{YZH}$ alleviates hepatic lipid accumulation. It also effectively restored elevated plasma levels of triglycerides (TG), total cholesterol (TC), alanine aminotransferase, and aspartate aminotransferase in HFD-fed mice. Mechanistically, these effects of YZH have associated with a decrease of AMPK/SREBP-1 pathway-mediated de novo lipogenesis and an increase of AMPK/ACC/CPT1A pathwaymediated mitochondrial fatty acid $\beta$ oxidation.

Conclusions: YZH supplementation ameliorated diet-induced obesity and hepatic steatosis by decreasing AMPK/SREBP-1 pathway-mediated de novo lipogenesis and increasing AMPK/ACC/CPT1A pathwaymediated mitochondrial fatty acid $\beta$ oxidation.
\end{abstract}

Keywords: Yin Zhi Huang (YZH); obesity; hepatic steatosis; de novo lipogenesis; mitochondrial fatty acid $\beta$ oxidation

Submitted Nov 01, 2019. Accepted for publication Dec 20, 2019.

doi: $10.21037 /$ atm.2020.01.31

View this article at: http://dx.doi.org/10.21037/atm.2020.01.31

\section{Introduction}

Obesity characterized as abnormalities in lipid metabolism represents a significant global health and socio-economic problem in the new millennium (1). In this setting, adipose tissues exceed its buffering capacity, resulting in lipid accumulation in the circulation and ectopic fat storage in organs like the liver (2). Non-alcoholic fatty liver disease (NAFLD), the primary cause of abnormal liver function in 
Western countries, is frequently associated with obesity (3). In addition to directly affecting liver function, NAFLD is often coexistent with features of metabolic syndrome, including type 2 diabetes mellitus, dyslipidemia, and hypertension. Its presence is an independent risk factor for the development of cardiovascular diseases (4). Nonalcoholic steatohepatitis (NASH), as an advanced form of NAFLD, will develop cirrhosis and end-stage liver diseases, including hepatocellular carcinoma (HCC). Unfortunately, no drugs have been approved for the treatment of NASH owing to its complicated pathophysiologic mechanism (5).

The development of NAFLD is closely associated with lipotoxicity caused by the storage of excessive free fatty acids (FFAs), which will induce the release of reactive oxygen species (ROS), as well as lead to oxidation stress, inflammation, apoptosis, and promoting hepatic injury. Considerable attention has been focused on reducing FFA levels in NAFLD treatment. Recently, traditional Chinese herbs have been reported to alleviate hyperlipidemia and NAFLD (6-10). Yin Zhi Huang (YZH), a traditional Chinese herbal formula, has been used in neonatal jaundice with apparent safety (11). It contains extracts from four different plants: Artemisia scoparia, Gardeniae fructus, Lonicerae Faponicae Flos, and Scutellaria baicalensis Georgi. In several regions of China, the former three are commonly used as foods. Several studies indicated that these four plants have anti-obesity or liver protection properties $(6-10,12,13)$. Thus, we proposed that YZH may be a promising strategy for NAFLD management.

When our body has too much fat either from foodderived fatty acid or from de-novo lipogenesis (DNL), which will synthesize fatty acids from excess carbohydrates, thus obesity occurs (14). The liver, as an essential organ for lipid metabolism, accumulates excessive fat, resulting in an imbalance of lipid between acquisition and consumption. Previous study demonstrated that the increase of liver lipid content in NAFLD patients is mainly attributable to the highly regulated metabolic pathway for the synthesis of fatty acids from acetyl-CoA or DNL (15). Firstly Acetyl-CoA is converted to malonyl-CoA by acetyl-CoA carboxylase (ACC), and malonyl-CoA is then changed to palmitate by fatty acid synthase (FAS). The transcriptional regulation of DNL is mainly controlled by sterol regulatory elementbinding protein 1 (SREBP-1), which is a transcriptional factor upregulating genes coding for ACC and FAS (16). The primary route for lipid disposal is through mitochondrial fatty acid $\beta$-oxidation (FAO), an essential process in which FFAs are esterified with CoA, transported into the mitochondria matrix, and oxidized to generate acetyl-CoAs (17). The transport of long-chain acyl-CoA esters into the mitochondria matrix is mediated by the carnitine palmitoyltransferase1A (CPT1A). CPT1A is considered as the rate-limiting enzyme for FAO and can be inhibited by malonyl-CoA, which is converted from acetylCoA by ACC (18). It is reported that AMP-activated protein kinase (AMPK) phosphorylation can suppress SREBP-1 cleavage processing and catalyze the phosphorylation of ACC to inactive ACC, thus increasing CPT1A level $(19,20)$. So, phosphorylation of AMPK can decrease DNL through AMPK/SREBP-1-mediated pathway and increase FAO through AMPK/ACC/CPT1A-mediated pathway.

In the present study, we investigated the effects of $\mathrm{YZH}$ on diet-induced obesity and hepatic steatosis and found that it can ameliorate body weight gain, hyperlipidemia, and hepatic steatosis. Mechanistically, YZH inhibited DNL in adipose tissue and liver via the AMPK/SREBP-1 pathway and increased FAO in the liver through the AMPK/ACC/ CPT1A pathway.

\section{Methods}

\section{YZH preparations}

YZH oral liquid was obtained from Beijing Shuanghe Gaoke Natural Medicine Co., Ltd. It contains four herbs, including $12 \mathrm{~g}$ Artemisia scoparia extract, $6.4 \mathrm{~g}$ Gardeniae fructus extract, $40 \mathrm{~g}$ Scutellaria baicalensis Georgi extract, and $8 \mathrm{~g}$ Lonicerae Faponicae Flos extract, which is dissolved in water to a final volume of $1 \mathrm{~L}$.

\section{Animal treatment}

Six-week-old C57BL/6J male mice (20-25 g) were purchased from Slack Laboratory Animal Co., Ltd (Shanghai, China). Mice were kept in an environmentally controlled room $\left(23 \pm 2{ }^{\circ} \mathrm{C}, 55 \% \pm 10 \%\right.$ humidity) with a 12 -hour light/dark cycle and allowed free access to food and water. Mice were randomly distributed into four groups with six animals each. The Chow group was given a normal chow diet (13.5\% of energy from fat; Slack Laboratory Animal Co., Ltd; China) and treated with normal saline by gavage at $30 \mathrm{~mL} / \mathrm{kg}$ daily. The HFD group was given a high-fat diet (HFD) $(60 \%$ of energy from fat; Research Diets D12492, USA) and treated with normal saline by gavage at $30 \mathrm{~mL} / \mathrm{kg}$ daily. The HFD $+\mathrm{L}$ and HFD $+\mathrm{H}$ group was given a HFD and treated with YZH by gavage at 10 or $30 \mathrm{~mL} / \mathrm{kg}$ daily, respectively. 
Food intake was recorded and calculated, as described previously (20). Body weight was monitored every week. At the end of the 16-week intervention period, animals fasted for 8 hours before sacrifice, and blood samples were taken from the inferior vena cava to analyze plasma biomarkers. Livers and adipose tissues were immediately collected, weighed, photographed, and stored for later analyze. The study was performed under the guiding principles for the care and use of laboratory animals approved by the Fudan University Animal Care Committee.

\section{Histopathological examinations}

Freshly isolated liver tissues and adipose tissues were fixed in $10 \%$ buffered formaldehyde solution, followed by dehydration and embedding in paraffin. Embedded tissues were cut into $8 \mu \mathrm{m}$ sections and stained with haematoxylin and eosin (H\&E). Liver steatosis was evaluated by two experienced pathologists blinded to experimental design protocol. The size of adipocytes was analyzed with Image-Pro Plus 6.0 (produced by Media Cybernetics Corporation, USA).

\section{Bodipy staining}

Bodipy staining was performed according to the standard procedures. Frozen liver tissue samples were sectioned and submerged in PBS for $10 \mathrm{~min}$ and then incubated in Bodipy Fl Dye (Thermo Fisher Scientific, Waltham, MA, USA) for $10 \mathrm{~min}$. The slides were then washed in PBS and immediately covered with DAPI (D9542; Sigma-Aldrich Corp. St. Louis, MO, USA) for nuclei staining. The sections were imaged using a Nikon Eclipse Ti inverted microscope (Nikon, Amstelveen, The Netherlands). Lipid accumulation was quantitatively analyzed with Image-Pro Plus 6.0 (Media Cybernetics Corporation, USA).

\section{Oil red $O$ staining}

Frozen liver sections ( $8-\mathrm{mm}$ thick) were stained with oil red O (cat. No. O0625, SigmaAldrich) according to the manufacturer's protocol. All stained sections were photographed using an inverted microscope under a $\times 200$ field (Nikon, Amstelveen, The Netherlands) and semiquantified by Image-Pro Plus 6.0 (Media Cybernetics Corporation, USA).

\section{Biochemical analysis}

Blood samples were obtained from the inferior vena cava and were centrifugated at 3,000 rpm for $5 \mathrm{~min}$ to obtain plasma. Plasma levels of triglyceride (TG), total cholesterol (TC), alanine transaminase (ALT), aspartate transaminase (AST), amylase, creatinine, and urea nitrogen were analyzed by Triglyceride kit, Total cholesterol kit (Kehua, Shanghai, China), AST kit, ALT kit, amylase kit, creatinine kit, and urea nitrogen kit (Nanjing Jiancheng Bioengineering Institute, Nanjing, China) respectively according to the manufacturer's instruction.

\section{Quantitative real-time reverse-transcription PCR}

Liver tissues were homogenized in TRIzol reagent (Invitrogen Life Technologies, Grand Island, NY), and total RNA was isolated according to the manufacturer's instructions and converted to cDNA with QuantiTect Reverse Transcription Kit (QIAGEN Gmbh, Hilden, Germany). Quantitative real-time reverse-transcription PCR (qRTPCR) was performed in triplicate using appropriate primers (Table S1), SYBR Premix Ex Taq (TaKaRa) and an ABI Prism 7500 Sequence Detection system (Applied Biosystems, Tokyo, Japan) with $\beta$-actin as control. Amplification was performed as follows: $10 \mathrm{~min}$ at $90{ }^{\circ} \mathrm{C}, 15 \mathrm{~s}$ at $95^{\circ} \mathrm{C}$ and $60 \mathrm{~s}$ at $60^{\circ} \mathrm{C}$ for 40 cycles. Relative gene expression was calculated using the $2-\Delta \Delta \mathrm{Ct}$ method.

\section{Immunobistochemistry}

The liver tissue was fixed in formalin, embedded with paraffin, and sliced into 4- $\mu \mathrm{m}$ sections. After being dewaxed, hydrated and subjected to heat-induced antigen retrieval, sections were incubated overnight at $4{ }^{\circ} \mathrm{C}$ with primary antibodies as follows: SREBP-1 mouse mAb (cat. No. sc13551, Santa Cruz Biotechnology), FAS mouse mAb (cat. No. sc-55580, Santa Cruz Biotechnology) and CPT1A rabbit pAb (cat. No. 15184-1-ap, Proteintech, USA). The sections were subsequently washed and incubated with HRP-conjugated anti-rabbit or anti-mouse secondary antibodies (cat. No. 111035-003, Jackson; cat. No. 115-035-003, Jackson). Finally, slides were treated with 3, 3'-diaminobenzidine (DAB) substrate and visualized with a Nikon Eclipse Ti inverted microscope (Nikon, Amstelveen, The Netherlands). The 
Page 4 of 13

immunostaining signaling was quantified with ImageJ 1.51 (NIH, Bethesda, Maryland, USA).

\section{Western blot analysis}

Liver and adipose tissue protein extraction and Western blotting were performed as described before (21). The primary antibodies were comprised of AMPK $\alpha$ rabbit mAb (cat. No. 5831, Cell Signaling, MA, USA), phosphoAMPK $\alpha$ rabbit mAb (cat. No. 50081, Cell Signaling, MA, USA), ACC rabbit mAb (cat. No. 3676, Cell Signaling, MA, USA, phospho-ACC rabbit mAb (cat. No. 11818, Cell Signaling, MA, USA), CPT1A rabbit pAb (cat. No. 15184-1-ap, Proteintech, USA), SREBP-1 mouse mAb (cat. No. sc-13551, Santa Cruz Biotechnology), peroxisome proliferation-activated receptor (PPAR)- $\alpha$ mouse mAb (cat. No. sc-398394, Santa Cruz Biotechnology) and FAS mouse mAb (cat. No. sc-55580, Santa Cruz Biotechnology). Primary antibody binding was detected using anti-rabbit or anti-mouse secondary antibodies conjugated with horseradish peroxidase (cat. No. 111-035-003, Jackson; cat. No. 115-035003, Jackson). The expression of antibody linked protein was determined by ECL ${ }^{\mathrm{TM}}$ Western Blotting Detection Reagents (Amersham Pharmacia Biotech Inc., NJ, USA). The optical density of the bands was quantified by Gel-Pro Analyzer 4 (Media Cybernetics Corporation, USA).

\section{Statistical analysis}

Data are presented as means \pm SEM. Differences in body weight were assessed using unpaired two-tailed Student's $t$-test. Data sets that involved more than two groups were assessed by one-way ANOVA followed by Newman-Keuls post hoc tests (see the legends of Figures 1-5). A P value $<0.05$ was considered to be statistically significant. In the figures, data with different superscript letters are significantly different based on post hoc ANOVA statistical analysis.

\section{Results}

\section{YZH blocks weight gain in mice fed with the HFD}

C57BL/6 mice fed with chow diet, HDF, or the combination of HFD and low/high dose YZH (low dose: $10 \mathrm{~mL} / \mathrm{kg}$ daily by gavage; high dose: $30 \mathrm{~mL} / \mathrm{kg}$ daily by gavage) for 16 weeks were used to test the effects of $\mathrm{YZH}$ on body weight. Compared to mice fed with chow diet, HDF fed mice showed an apparent increase in the body
Yao et al. Yin Zhi Huang ameliorates obesity and hapatic steatosis

weight over the test period (Figure $1 A, B$ ) companied with visceral fat mass and adipocyte diameter measured by $\mathrm{H} \& \mathrm{E}$ staining (Figure $1 D, E, F, G, H, I$ ), which were significantly reduced by adding $\mathrm{YZH}$ (Figure $1 A, B, D, E, F, G, H, I$, Table S2) in a dose-dependent manner. Notably, the food intakes were almost equal in each group (Figure 1C), suggesting that the $\mathrm{YZH}$-induced anti-obesity role is independent of food consumption.

\section{YZH ameliorates hepatic steatosis in HFD-induced obese mice}

Obesity, especially visceral obesity, is often related to hepatic steatosis. Consistently, HFD-fed mice showed an increased liver weight (Figure 2A,E, Table S2) and abundant hepatic steatosis compared to mice with chow diet, as indicated by gross morphology and histological examination using $\mathrm{H} \& \mathrm{E}$ staining, oil red $\mathrm{O}$ staining and bodipy staining. Large lipid droplets were observed in the liver of HFD-fed mice, while adding $\mathrm{YZH}$ significantly compromised liver weight gain and hepatic lipid droplets in a dose-dependent manner (Figure 2A,B,C,D,E,F,G,H, Table S2). Together, these results implicated that $\mathrm{YZH}$-associated diet reduces the hepatic steatosis in HFD-fed mice.

\section{Effect of $Y Z H$ on plasma lipid parameters and liver aminotransferase levels and safety of $\mathrm{YZH}$ on biochemical indexes}

Obesity, usually characterized by hepatic steatosis, is associated with aberrant levels of lipid and cholesterol, and increased aminotransferases. Here we observed an increased level of plasma TG induced in HFD-fed mice, as compared to that in chow-fed mice, adding a high dose of $\mathrm{YZH}$ significantly blocked the increase (Figure 3 A, Table S2), while a low dose of $\mathrm{YZH}$ observed mild effect. It is evident that feeding with HDF causes a high amount of cholesterol. However, a high dose of $\mathrm{YZH}$ clearly neutralized this effect (Figure 3B, Table S2).

HFD-mediated liver injury has been reported previously (22). We interestingly found that $\mathrm{YZH}$ protected the liver in this context indicated by reduced levels of ALT and AST (Figure 3C,D, Table S2). Additionally, we examined plasma creatinine, urea nitrogen and amylase, and no significant differences were found among groups (Figure 3E,F,G, Table S2). These results indicated that a good tolerance of $\mathrm{YZH}$ in mice, which is consistent with the results in human (11). Moreover, no 
A

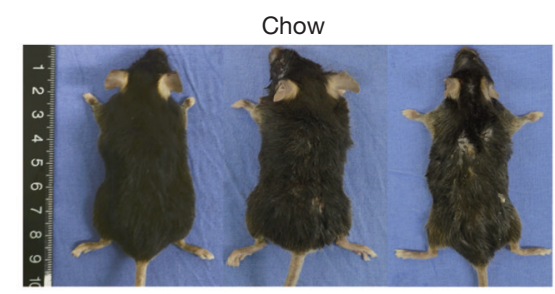

HFD+L

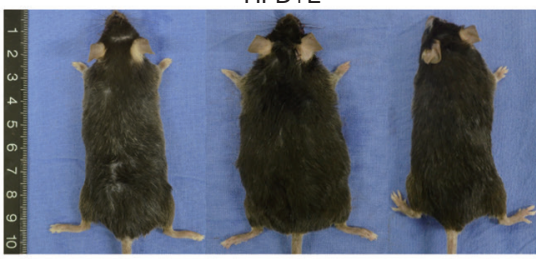

D
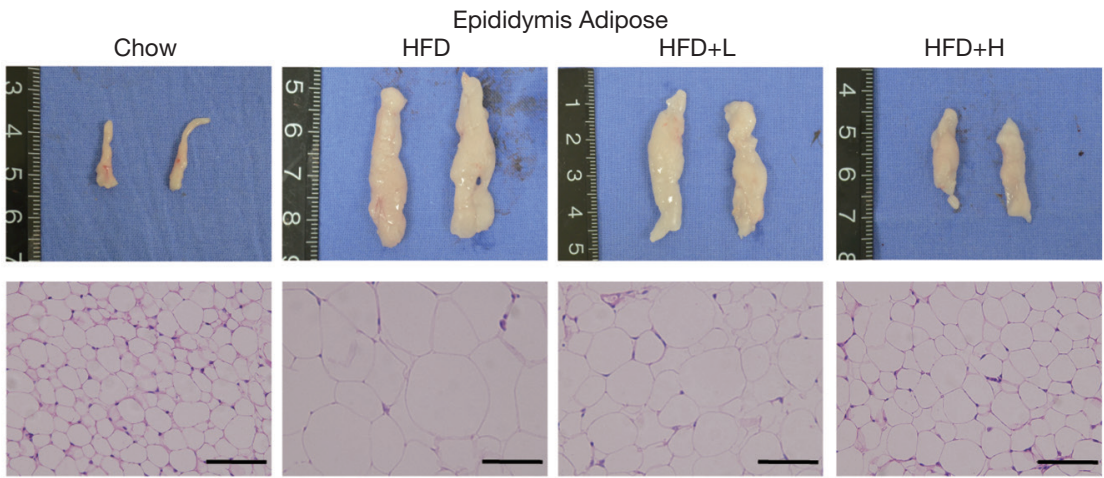

G
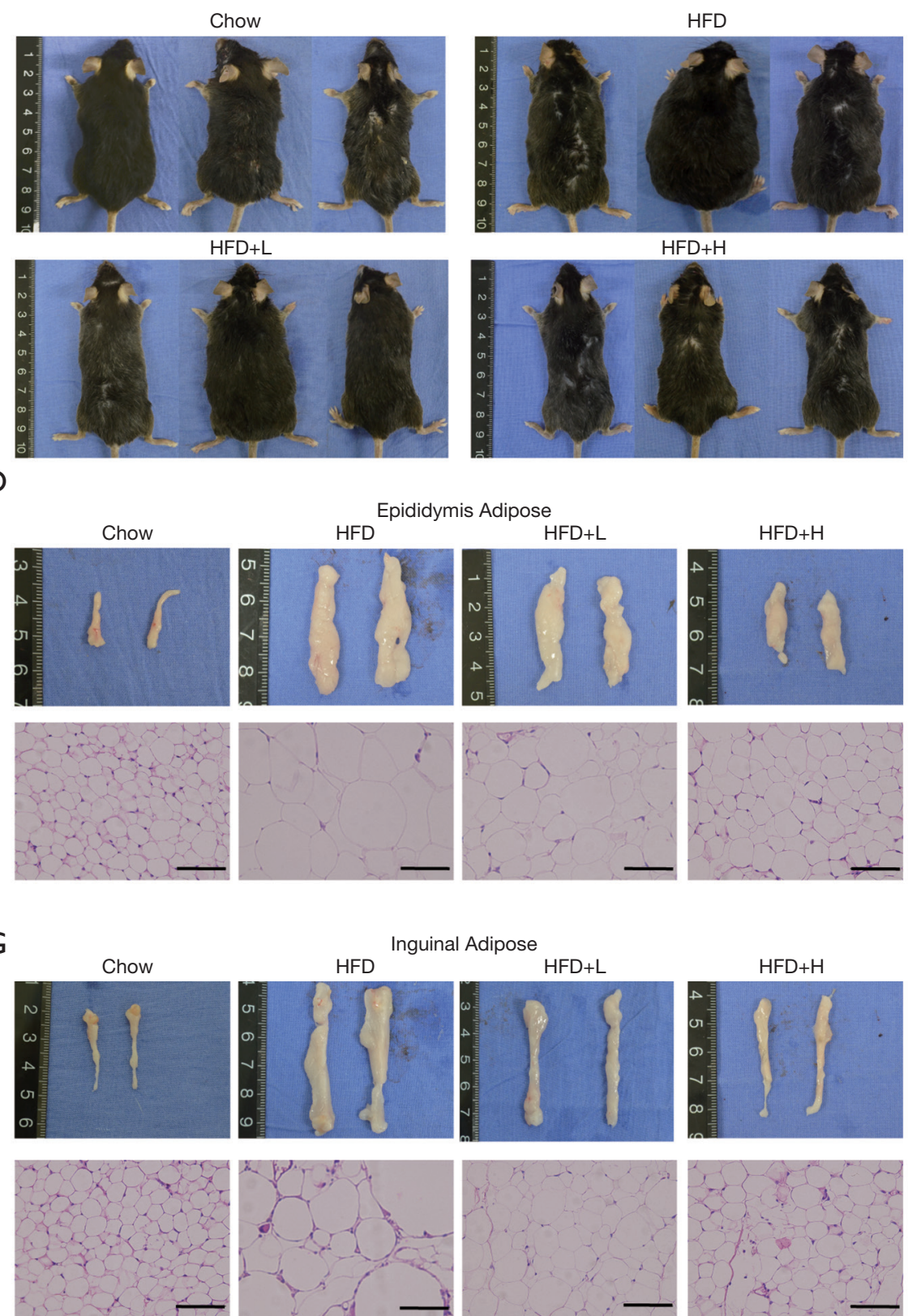

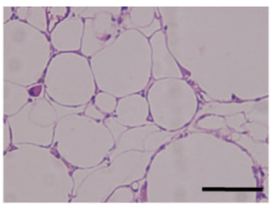

HFD+L
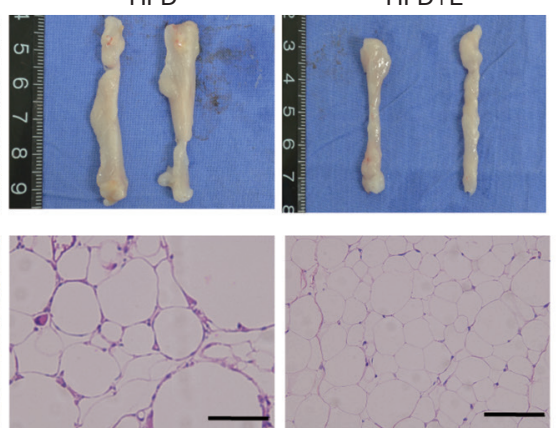

$\mathrm{F}$

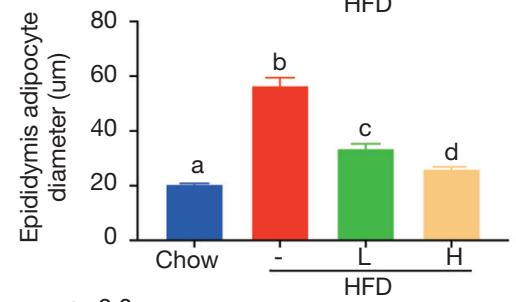

$\mathrm{H}$

B
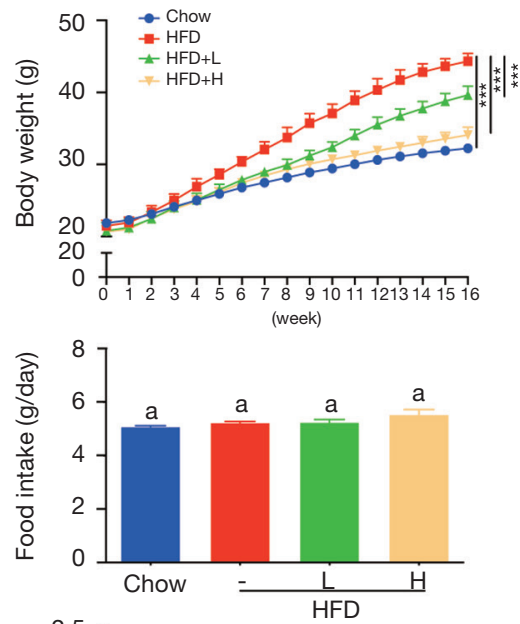

E
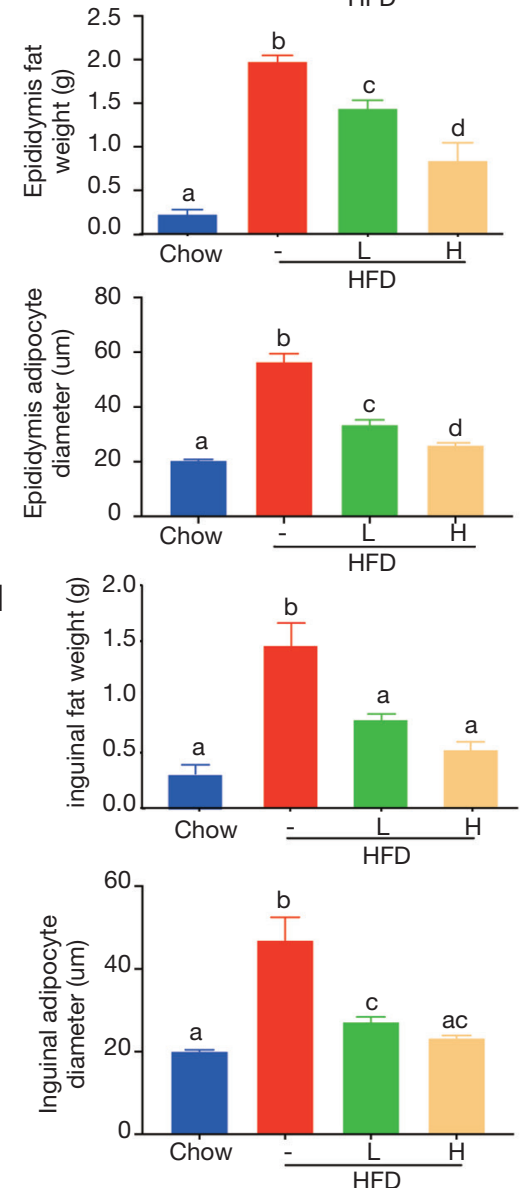

Figure 1 Effect of YZH on body weight. Mice were fed HFD supplements with or without YZH (low dose and high dose) for 16 weeks. Chow group-administered normal diet as a vehicle. At the end of the experiment, all mice of each group were killed by sodium pyridobatosa abdominal injections. (A) Representative mice photographs of each group were shown after aneasthesia; (B) body weight was calculated weekly; (C) food intake; (E) epididymal fat weight; (F) epididymal adipocytes diameter; (H) inguinal fat weight; (I) inguinal adipocytes diameter of each group were quantified carefully; $(\mathrm{D}, \mathrm{G})$ representative gross morphology and histology of epididymal and inguinal adipose tissues by H\&E staining were displayed visually. Data were expressed as mean \pm S.E.M ( $\mathrm{n}=6$ per group). Scale bar: $100 \mu \mathrm{m}$. Body differences in B were analysed with unpaired two-tailed Student's $t$-test $\left.{ }^{* * *} \mathrm{P}<0.001\right)$. Graph bars in $\mathrm{C}, \mathrm{E}, \mathrm{F}, \mathrm{H}$, and I marked with different letters on top represent statistically significant results $(\mathrm{P}<0.05)$ based on Newman-Keuls post hoc one-way ANOVA analysis, whereas bars labeled with the same letter means results that show no statistically significant differences. YZH, Yin Zhi Huang; HFD, high-fat diet. 

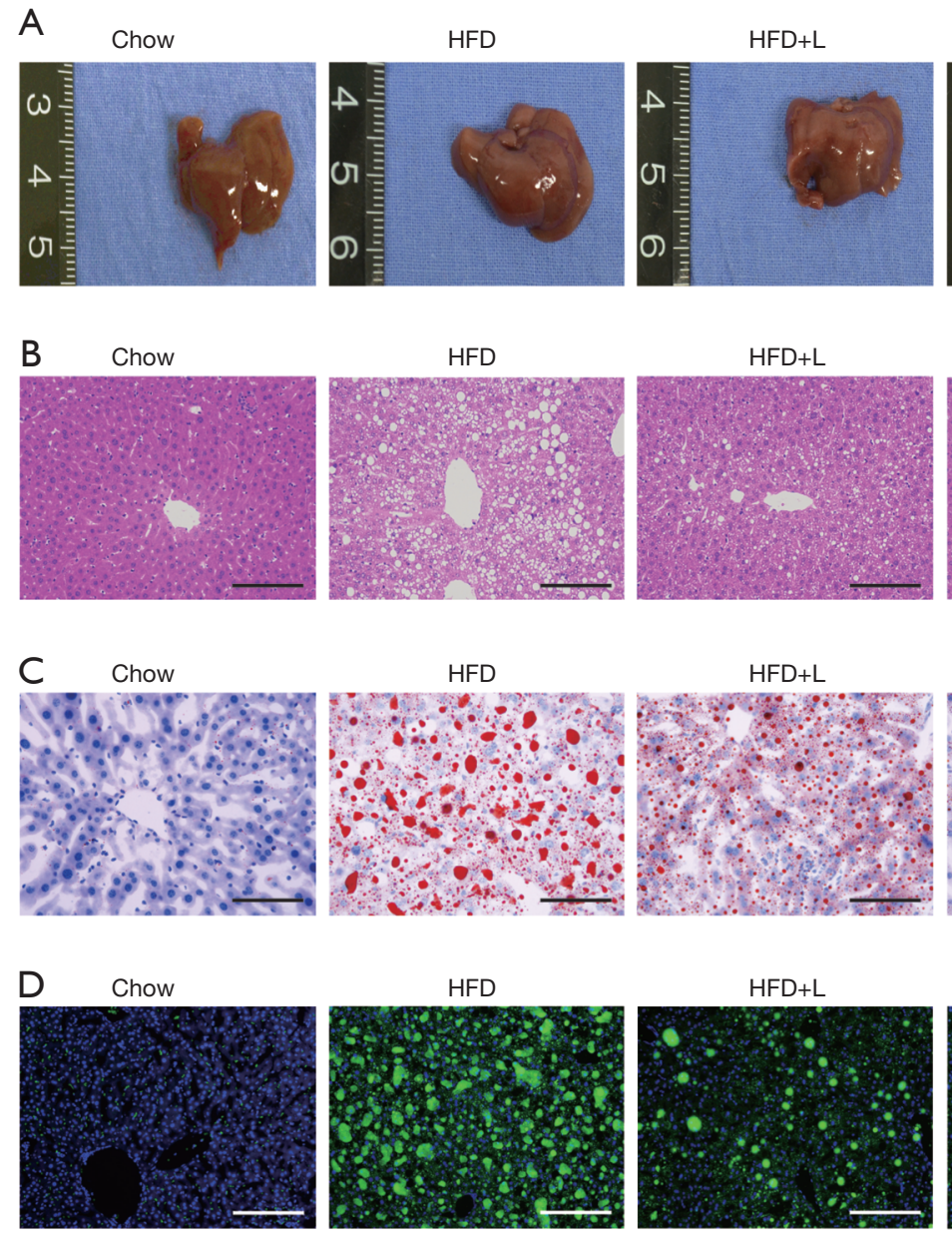
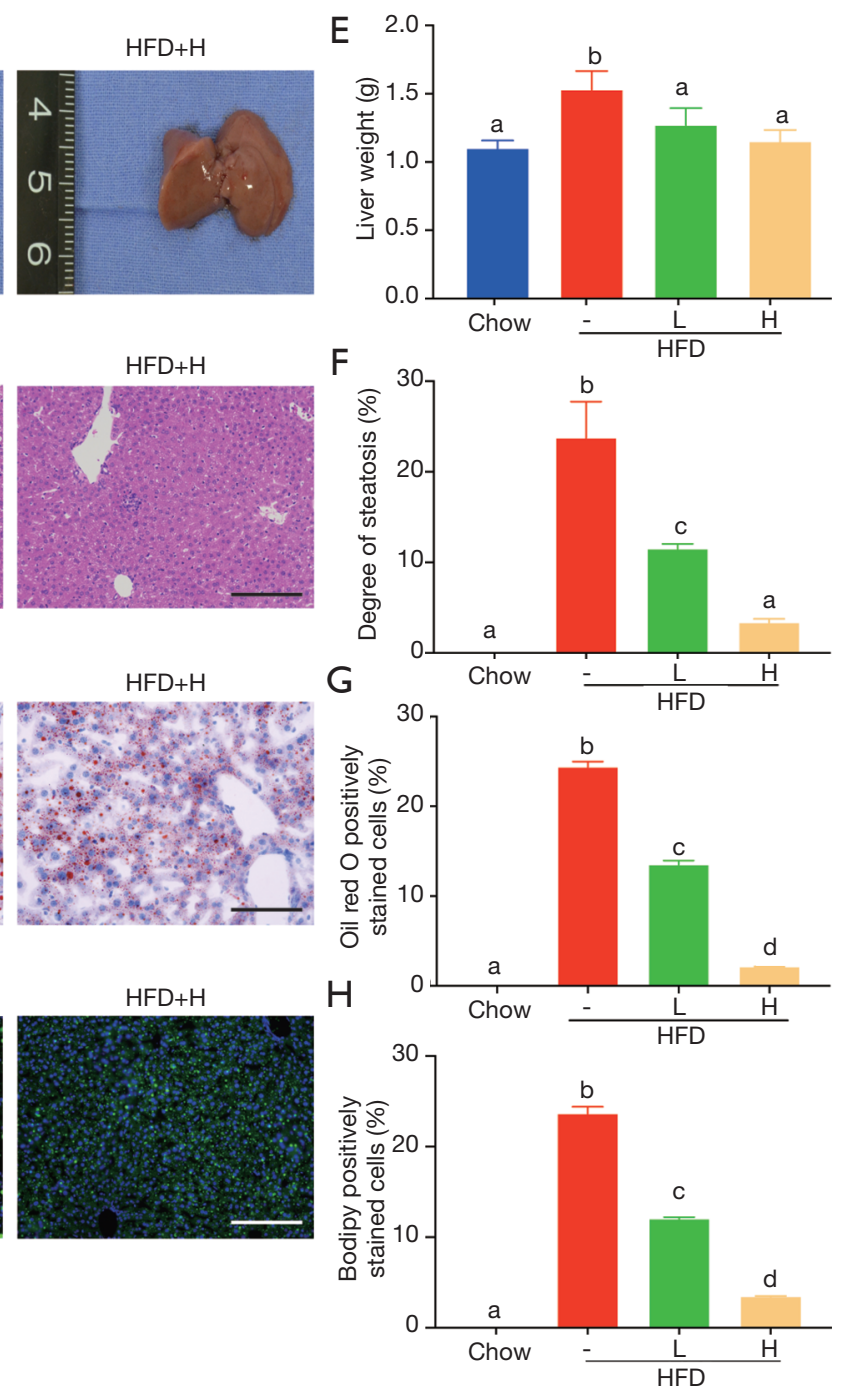

Figure 2 Effects of $\mathrm{YZH}$ on hepatic steatosis in HFD-fed mice. Chow- and HFD-fed mice were treated daily with normal saline or YZH at 10 or $30 \mathrm{~mL} / \mathrm{kg}$ by intragastric gavage for 16 weeks ( $\mathrm{n}=6$ for each group). (A) The gross morphology of livers; (B) representative histological images of H\&E-stained sections (×200); (C) oil Red O-stained sections ( $\times 200)$; and (D) bodipy-stained sections ( $\times 100)$ of each group were shown. YZH significantly decreased (E) liver weight, (F) degree of steatosis, $(\mathrm{G})$ oil red O-stained cells, and (H) bodipy fluorescence intensity. Data were presented as mean \pm S.E.M (n=6 per group). Scale bar: $100 \mu \mathrm{m}$ for (B) and (C); Scale bar: $200 \mu \mathrm{m}$ for (D). Graph bars with different letters on top represent statistically significant results $(\mathrm{P}<0.05)$ based on Newman-Keuls post hoc one-way ANOVA analysis, whereas bars with the same letter correspond to results that show no statistically significant differences. YZH, Yin Zhi Huang; HFD, highfat diet. 

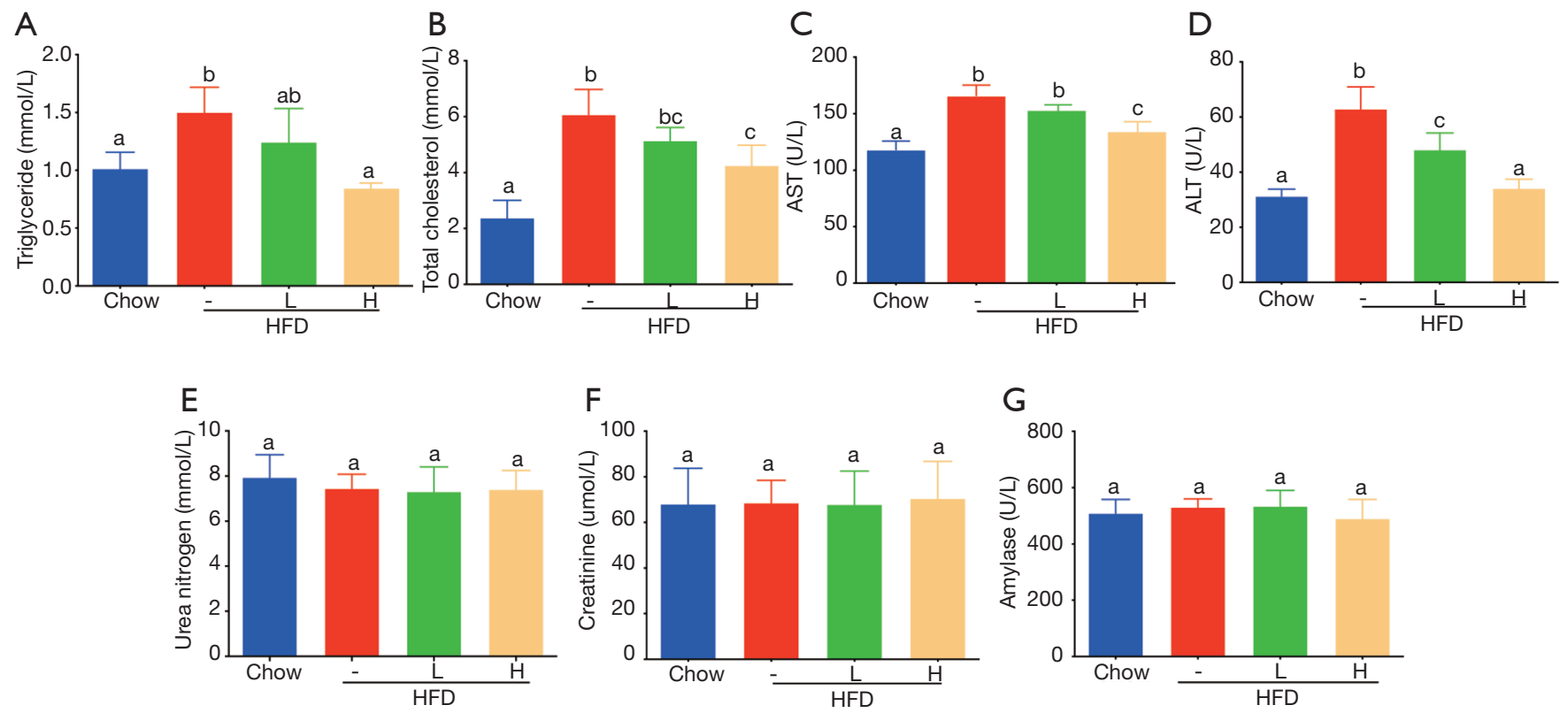

Figure 3 Effect of $\mathrm{YZH}$ on plasma lipid parameters and liver aminotransferase levels and safety of YZH to kidney and pancreas. (A) YZH reduced triglycerides (TG), (B) total cholesterol (TC), (C) aspartate aminotransferase (AST), and (D) alanine aminotransferase (ALT) levels in HFD-fed mice. Otherwise, YZH did not affect plasma creatinine, urea nitrogen, and amylase (E-G). Data were presented as mean \pm S.E.M ( $\mathrm{n}=6$ per group). Graph bars with different letters on top represent statistically significant results $(\mathrm{P}<0.05)$ based on Newman-Keuls post hoc one-way ANOVA analysis, whereas bars with the same letter correspond to results that show no statistically significant differences. YZH, Yin Zhi Huang.

toxicity was observed in hepatic cell line L02 incubating with different concentrations of YZH for either 24 or $48 \mathrm{~h}$ (Figure S1).

\section{$Y Z H$ decreased DNL through activating AMPK/SREBP-1 pathway and increased $F A O$ via activating AMPK/ACC/ CPT1A pathway}

Since food intake among HFD group and HFD with either low dose or high dose of $\mathrm{YZH}$ groups had no significant difference, we speculated that the anti-obesity and antihepatic steatosis effects of $\mathrm{YZH}$ might involve in lipid metabolism. We screened genes involved in fatty acid synthesis, transport, $\beta$-oxidation, bile acid, and cholesterol metabolism in the liver. The results showed that $\mathrm{YZH}$ had no significant effect on the level of the genes including 3-hydroxy-3-methylglutaryl CoA reductase (Hmgcr), fatty acid-binding protein 1 (Fabp1), long-chain acyl-CoA dehydrogenase (Lcad), lipoprotein lipase (Lpl), Cholesterol 7a-hydroxylase (Cyp7a1), and Cytochrome P450 Family 2 Subfamily E Member 1 (CYP2E1) (Figure S2). SREBP-1 and FAS, which can be inhibited by pAMPK, are essential proteins facilitating DNL, and CPT1A is the rate-limiting enzyme in mitochondrial FAO and can be increased by pAMPK. Next, we tested the expression of these proteins. The results demonstrated that YZH decreased SREBP-1 and FAS, along with increased CPT1A both in mRNA and protein levels in liver tissues (Figure 4A, B, C,D,E, F, G, H,I). In the adipose and the liver tissue, $\mathrm{YZH}$ increased the pAMPK/AMPK ratio and decreased SREBP-1 and FAS levels to diminish DNL (Figure $5 A, B$ ). Moreover, YZH increased pACC/ACC ratio and raised CPT1A and PPAR- $\alpha$ protein levels to enhance FAO in the liver (Figure 5C,D,E), although the mRNA level of PPAR- $\alpha$ was inconsistent with its protein level (Figure S2). These results indicate that YZH may decrease AMPK/SREBP-1 pathway-mediated DNL and increase AMPK/ACC/CPT1A pathway-mediated mitochondrial FAO.

\section{Discussion}

There has been an explosive growth of interest in obesity and NAFLD because of their global prevalence. Until recently, no drugs have been approved for the treatment of 
A
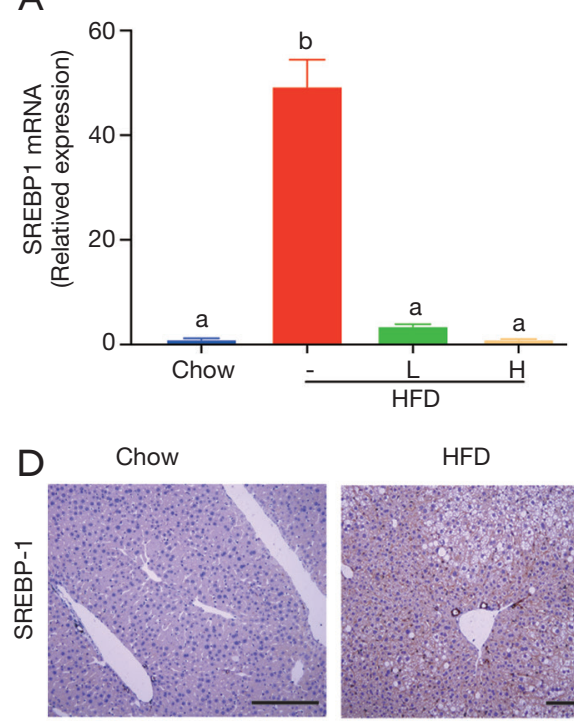

HFD

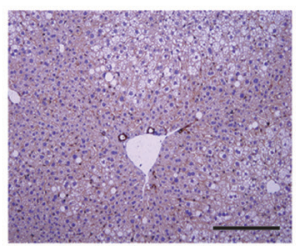

$\mathrm{F}$
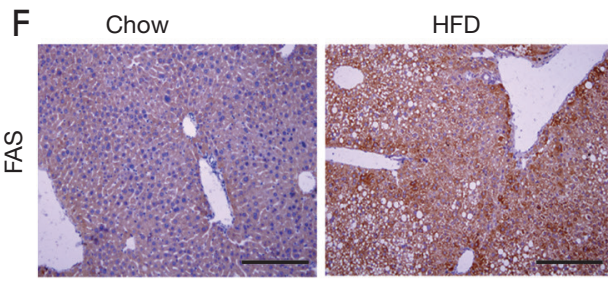

$\mathrm{H}$

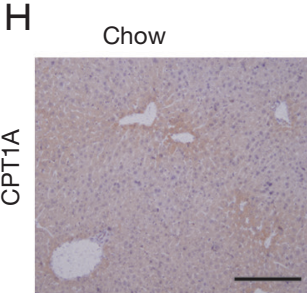

B

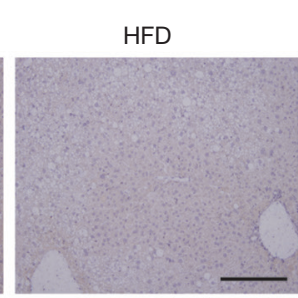

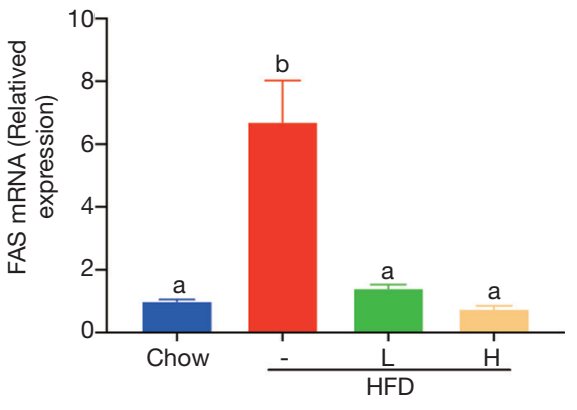

HFD+L
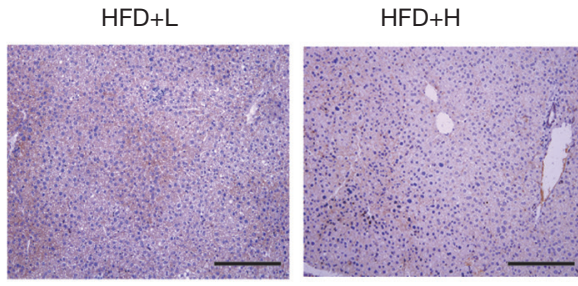

HFD+L
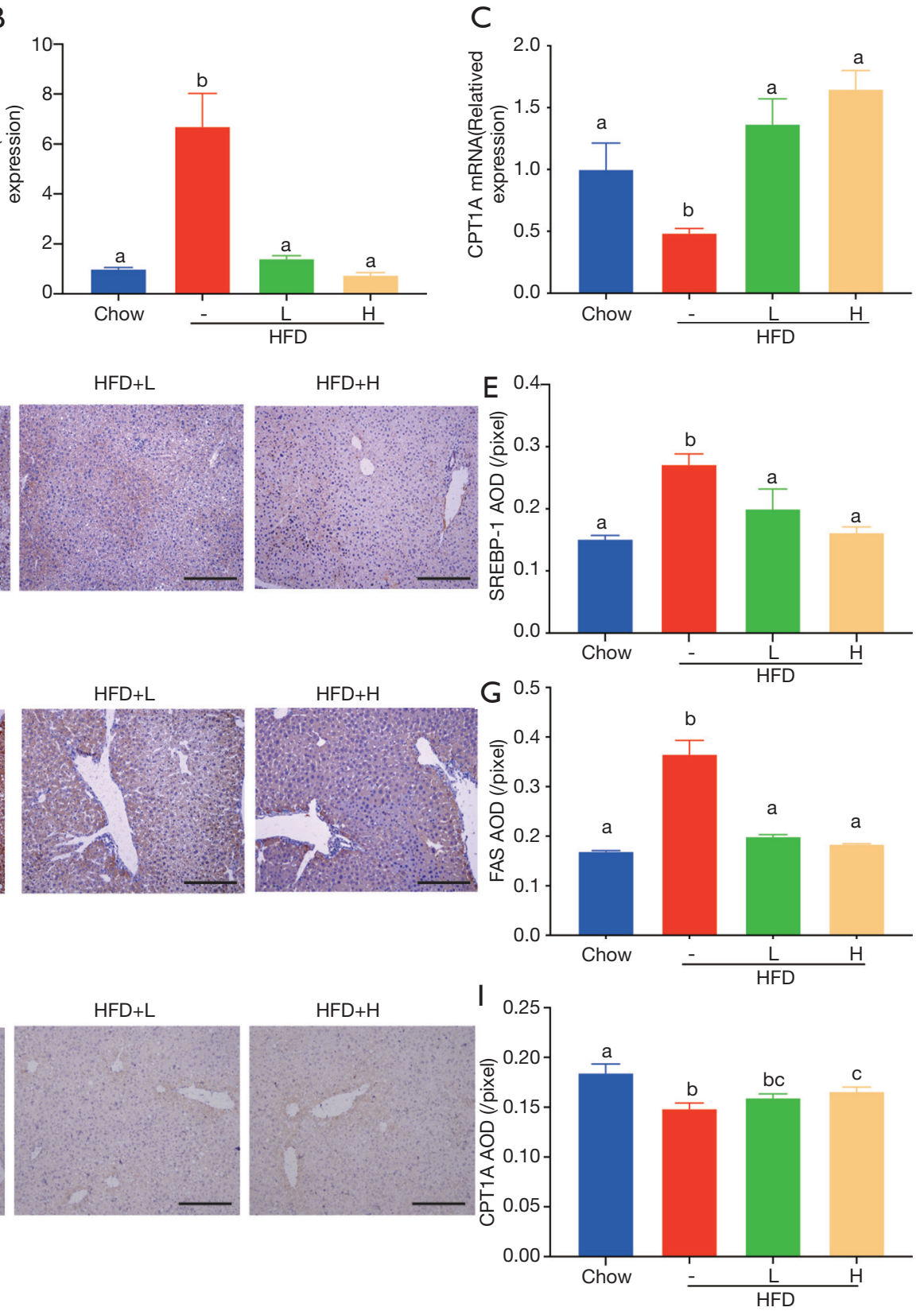

Figure 4 Effect of YZH on SREBP-1, FAS, and CPT1A levels in the liver. The mRNA levels of SREBP-1, FAS, and CPT1A in the liver were analyzed by Real-time PCR (A-C). The protein levels of SREBP-1, FAS, and CPT1A in the liver were evaluated by immunohistochemistry (D-I). Data were presented as mean \pm S.E.M ( $\mathrm{n}=6$ per group). Scale bar: $200 \mu \mathrm{m}$. Graph bars with different letters on top represent statistically significant results $(\mathrm{P}<0.05)$ based on Newman-Keuls post hoc one-way ANOVA analysis, whereas bars with the same letter correspond to results that show no statistically significant differences. YZH, Yin Zhi Huang. 
A

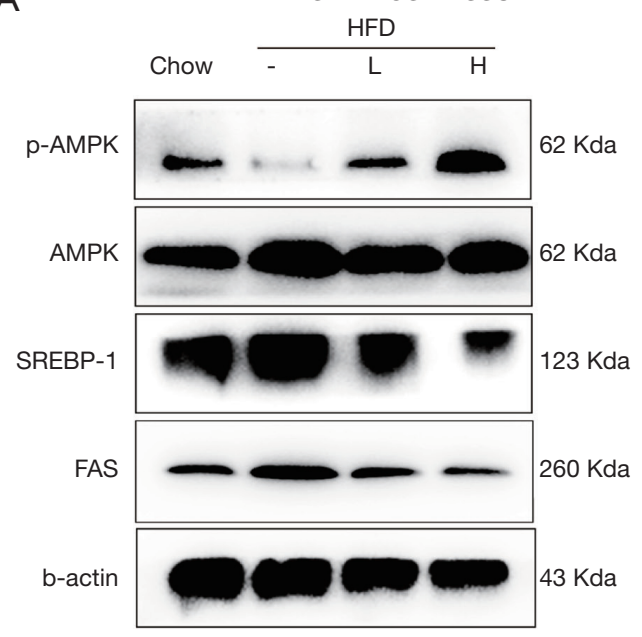

C

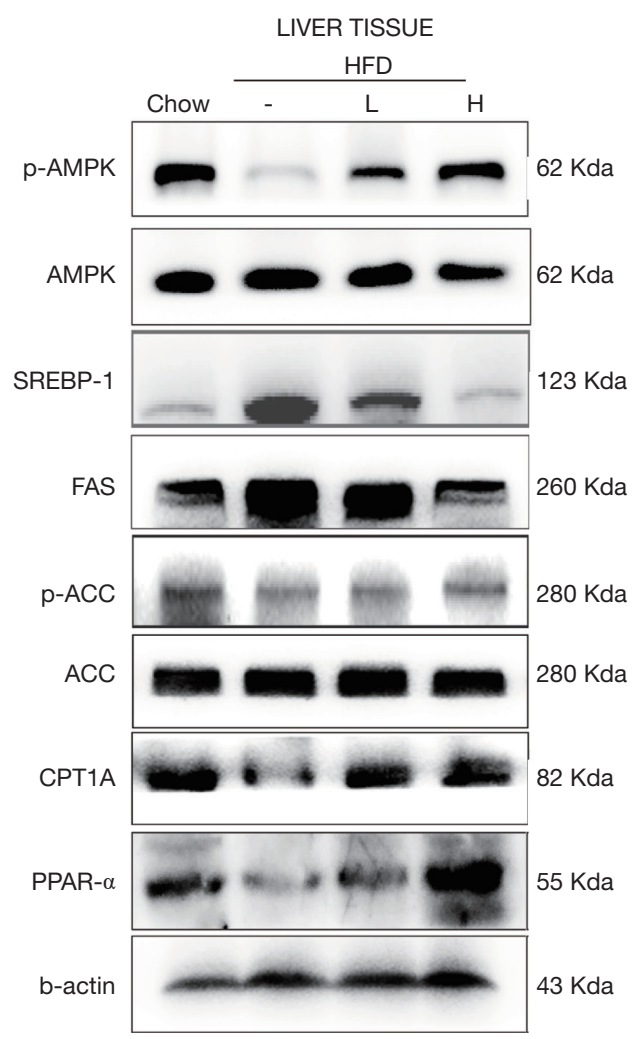

B
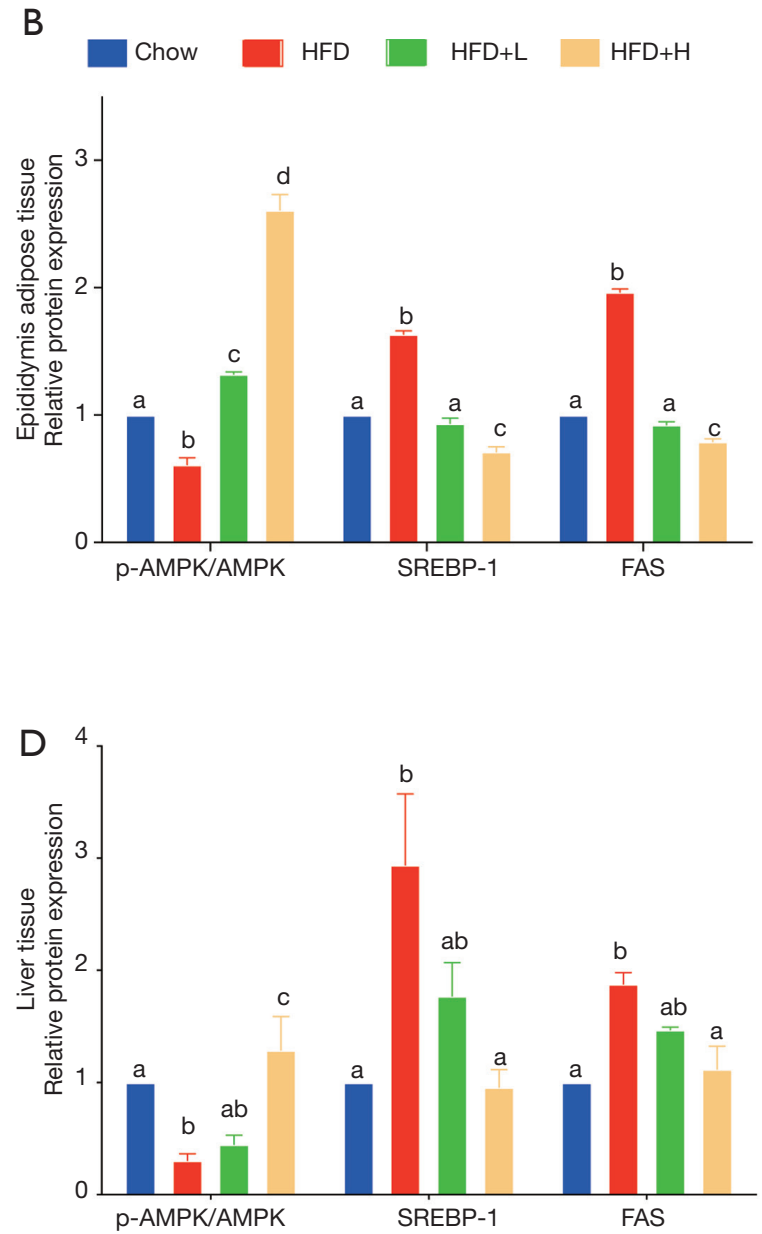

$E$

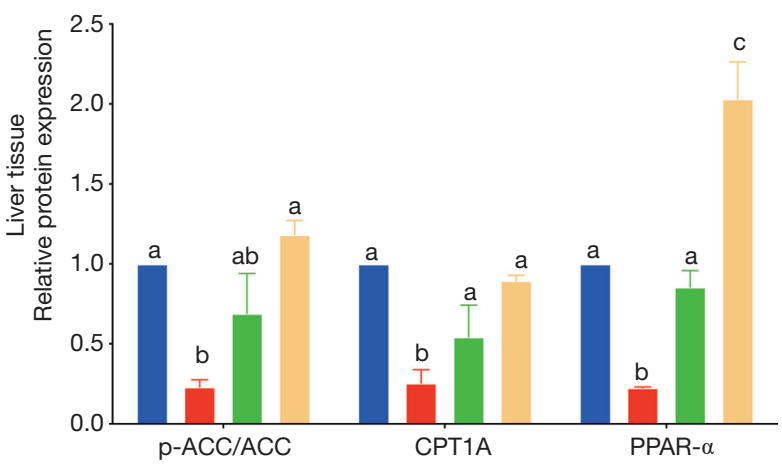

Figure 5 YZH regulated DNL through AMPK/SREBP-1 pathway and FAO via AMPK/ACC/CPT1 pathway. AMPK, pAMPK, SREBP-1, and FAS protein levels in liver and epididymis adipose tissue together with ACC, pACC, CPT1A, and PPAR- $\alpha$ protein levels in liver were analyzed by Western blotting $(\mathrm{A}, \mathrm{C})$. The relative protein levels were expressed as the fold in comparison to the Chow group after normalized to $\beta$-actin (B,D,E). The values were presented as mean \pm S.E.M. ( $\mathrm{n}=6$ per group). Graph bars with different letters on top represent statistically significant results $(\mathrm{P}<0.05)$ based on Newman-Keuls post hoc one-way ANOVA analysis, whereas bars with the same letter correspond to results that show no statistically significant differences. YZH, Yin Zhi Huang. 


\section{Page 10 of 13}

NAFLD (5). Combined therapies may be useful, including lipid-lowering drugs, such as statins. However, one of the most common side effects of statins is liver damage. Therefore, developing an anti-obesity and anti-NAFLD agent with hepatoprotective properties will be favorable to these obese patients. YZH is a decoction that has been used for centuries in Asia to treat neonatal jaundice, and also has been widely used as a hepatoprotective drug (23). In the present study, we clarified that YZH could reduce obesity and hepatic steatosis in a dose-dependent manner in HFDfed mice. Moreover, adipose mass, the size of adipocytes, plasma TG, TC, and transaminases were also decreased by feeding with $\mathrm{YZH}$, suggesting that $\mathrm{YZH}$ might be an ideal agent for patients with obesity or NAFLD.

YZH contains extracts from Artemisia scoparia, Gardeniae fructus, Scutellaria baicalensis Georgi, and Lonicerae Faponicae Flos, three of the four components are eaten as foods in some area of Asia. As a drug for neonatal jaundice, YZH oral liquid had no side effects reported (11). In our study, we observed no liver, kidney, or pancreas damage in mice despite administration at about 30 times the human dose and no cell toxicity in vitro with $\mathrm{YZH}$ at volume concentration from $1 \%$ to $20 \%$. The extracts of Artemisia scoparia extracts had been reported to have protection on islet $\beta$ cell to release diabetes mellitus and to have lipid-lowering effect accompanied by alleviating NAFLD (24). The further report demonstrated that Artemisia scoparia extracts composed of scoparone and flavonoids, which were found in foods with an antiobesity ability (25). Gardeniae Fructus is widely used in Asian countries as a complementary and alternative therapy. The main components of Gardeniae Fructus comprise geniposide and crocin exhibiting anti-diabetes and hypolipidemic activities $(26,27)$. In spontaneously obese type 2 diabetic mice, geniposide diminished body weight and visceral fat accumulation, alleviated abnormal lipid metabolism, and suppressed intrahepatic lipid accumulation.

Furthermore, geniposide showed relief of abnormal glucose tolerance and hyperinsulinemia (26). In dietinduced hyperlipidemic rats, a 10-day treatment with crocin largely reduced serum TG, TC, low-density lipoprotein cholesterol, and very-low-density lipoprotein cholesterol levels in a daily dose range of $25-100 \mathrm{mg} / \mathrm{kg}$ (27). Genipin, an intestinal bacterial metabolite of geniposide, was also showed to mitigate HFD-induced obesity, hyperlipidemia, and hepatic lipid accumulation $(7,8)$. Lonicerae Faponicae Flos, as one of the most famous traditional Chinese medicines, has been applied in healthcare in Asian countries for a long time. Besides, it can be used as functional foods, cosmetics, and other applications in some areas of Asia with anti-inflammatory, antibacterial, lipid-lowering, and hepatoprotective functions $(12,13,28)$. As mentioned above, both two components are listed in the 2008 Chinese catalog for the homology of medicine and food, and it also suggests that YZH may have high safety. Scutellaria baicalensis has a long history of usage in traditional Chinese medicine, and its raw extracts were displayed to have a robust lipidlowering effect (9). It contains diverse flavonoids, which are the main active compounds (29). Among them, the most representative ingredients are baicalin, baicalein, wogonoside, and wogonin (30). By decreasing lipogenesis and increasing fatty acid oxidation, baicalin played a remarkable role in anti-obesity and anti-steatosis activities in rodents $(31,32)$.

To determine the potential mechanisms by which $\mathrm{YZH}$ prevents HFD-induced obesity and hepatic steatosis, we investigated genes related to DNL and FAO. SREBP-1 is a crucial transcription factor of DNL and upregulates expression of downstream molecules, including ACC and FAS, to promote adipogenesis. Furthermore, the cleavage, nuclear translocation, and transcriptional activity of SREBP-1 are negatively regulated by phosphorylated AMPK (33). Herein, our results manifested that YZH elevated the phosphorylation of AMPK and downregulated protein levels of SREBP-1 and FAS to weaken DNL in the liver and adipose tissues. Consistent with our findings, Artemisia scoparia extracts were found to attenuate SREBP-1 and FAS expression considerably and to raise the ratio of pACC/ACC in the liver of mice when compared to HFD group (6). Genipin, a primary active ingredient of Gardenia jasminoides Ellis, was confirmed to alleviate hyperlipidemia and lessen lipid accumulation in the liver of HFD-fed obese mice through inhibiting HFD feedinginduced upregulation of hepatic protein levels of SREBP-1, FAS, and SCD1 in a dose-dependent manner (7). Baicalin, one of the major flavonoids in Scutellaria baicalensis, suppressed body weight gain, decreased the elevated serum FFA, and reduced hepatic lipid accumulation by enhancing the phosphorylation of AMPK and ACC, and by downregulating SREBP-1 and FAS in HFD-fed rats and HepG2 cells (10).

In the process of mitochondrial FAO, fatty acid transports into the mitochondria relying on CPT1A, which is considered as the rate-limiting enzyme for FAO. MalonylCoA is a native inhibitor of CPT1A and is converted from acetyl-CoA by ACC. ACC is phosphorylated by AMPK at Ser79 to be inactivated and then lowers the malonyl-CoA 
level to boost FAO with increased CPT1A (34). In our study, we found that $\mathrm{YZH}$ activated AMPK to phosphorylate ACC inactivating its' ability to convert malonyl-CoA from acetylCoA, consequently increased CPT1A content to accelerate mitochondrial FAO. Congruent with previous research on Artemisia scoparia extracts, the lipid-lowering effect was found in HFD mice supplement with Artemisia scoparia extracts via increasing mitochondrial FAO by facilitating the activity of CPT1A as compared with HFD control group (24). With quantitative chemoproteomic profiling, previous research found that baicalin also directly activated hepatic CPT1A to promote the lipid influx into mitochondria for oxidation (32). The protein level of PPAR- $\alpha$ in the liver was increased by $\mathrm{YZH}$ compared with the HFD-diet group, while the mRNA levels were inconsistent. Course there were many complicated and varied post-transcriptional mechanisms involved in turning mRNA into protein (35). Furthermore, how YZH, actually its' active ingredients, influences PPAR- $\alpha$ needs further research.

In summary, our study uncovered $\mathrm{YZH}$, most of which are from foods and have excellent safety, effectively antagonized HFD-induced hyperlipidemia and hepatic steatosis. Such effects were achieved by decreasing DNL through AMPK/SREBP-1 pathway and increasing mitochondrial FAO through AMPK/ACC/CPT1A pathway. Hence, we choose $\mathrm{YZH}$ as an excellent candidate for people to relieve obesity and related metabolic syndromes instead of active ingredients, such as genipin or baicalin. Course $\mathrm{YZH}$ has been used for centuries in Asia to treat neonatal jaundice and has proper safety. In China, it is produced by famous state-owned enterprises with reasonable quality control. Intriguingly, traditional Chinese medicine formulas usually contain several herbs based on the principle of "Jun-Chen-Zuo-Shi". "Jun" means the emperor treating the leading cause of the disease, such as Artemisia scoparia in YZH. "Chen" means minister increasing the actions of "Jun" or treating concomitant symptoms, such as Gardeniae Fructus in YZH. "Zuo" means assistant reducing or abolishing possible toxic effects of the Jun or Chen herbs and also treating accompanied symptoms, such as Lonicerae faponicae Flos in YZH, and "Shi" means emissary delivering or guiding other herbs to the target organs, such as Scutellaria baicalensis Georgi in $\mathrm{YZH}$. In fact, pharmacokinetics analysis also corroborated that baicalin increased the absorption of geniposide (36).

Additionally, $\mathrm{YZH}$ is contributed to supporting the elimination of intravenously infused bilirubin via the constitutive androstane receptor (CAR, NR1I3) (37). It is also found that CAR is highly expressed in the liver and intestine, and its' activation is validated to ameliorate diabetes and fatty liver disease $(38,39)$. The potential mechanism of $\mathrm{YZH}$ alleviating obesity and hepatic steatosis may be based on CAR-mediated bile acid clearance or orchestrating gut microbiota. Future efforts are still required to elucidate the precise mechanisms of $\mathrm{YZH}$. Though we demonstrated that YZH could facilitate the phosphorylation of AMPK to decrease DNL and increase mitochondrial FAO, consistent with previous research on Artemisia scoparia, Gardeniae fructus and Scutellaria baicalensis Georgi $(6,31,40)$, the direct evidence was not included. Further study would add a group of HFD + YZH + compound C, a well-known AMPK inhibitor.

\section{Conclusions}

YZH supplementation ameliorated diet-induced obesity and hepatic steatosis, in company with lipid-lowering effect, liver protection, and no side effect. The underlying mechanism involved decreasing AMPK/SREBP-1 pathwaymediated DNL and increasing AMPK/ACC/CPT1A pathway-mediated mitochondrial FAO. In light of the apparent effect of $\mathrm{YZH}$ on obesity, together with hepatic steatosis and the complicated pathophysiologic mechanism of NAFLD, more work will be needed in the near future.

\section{Acknowledgments}

We thank Jimin Zhu for providing technical help, editing manuscript's language and giving advices for arranging figures.

Funding: This research was funded by the National Natural Science Foundation of China ([grant numbers: 81500460) and Zhongshan Hospital Foundation (grant number: 2017ZSYQ11).

\section{Footnote}

Conflicts of Interest: The authors have no conflicts of interest to declare.

Ethical Statement: The authors are accountable for all aspects of the work in ensuring that questions related to the accuracy or integrity of any part of the work are appropriately investigated and resolved. This study has been approved by the Ethics Committee of Zhongshan Hospital Fudan University. 


\section{Page 12 of 13}

Open Access Statement: This is an Open Access article distributed in accordance with the Creative Commons Attribution-NonCommercial-NoDerivs 4.0 International License (CC BY-NC-ND 4.0), which permits the noncommercial replication and distribution of the article with the strict proviso that no changes or edits are made and the original work is properly cited (including links to both the formal publication through the relevant DOI and the license). See: https://creativecommons.org/licenses/by-nc-nd/4.0/.

\section{References}

1. Wolfenden L, Ezzati M, Larijani B, et al. The challenge for global health systems in preventing and managing obesity. Obes Rev 2019;20 Suppl 2:185-93.

2. Canfora EE, Meex RCR, Venema K, et al. Gut microbial metabolites in obesity, NAFLD and T2DM. Nat Rev Endocrinol 2019;15:261-73.

3. Milić S, Lulic D, Stimac D. Non-alcoholic fatty liver disease and obesity: biochemical, metabolic and clinical presentations. World J Gastroenterol 2014;20:9330-7.

4. Adams LA, Anstee QM, Tilg H, et al. Non-alcoholic fatty liver disease and its relationship with cardiovascular disease and other extrahepatic diseases. Gut 2017;66:1138-53.

5. Friedman SL, Neuschwander-Tetri BA, Rinella M, et al. Mechanisms of NAFLD development and therapeutic strategies. Nat Med 2018;24:908-22.

6. Wang ZQ, Zhang XH, Yu Y, et al. Artemisia scoparia extract attenuates non-alcoholic fatty liver disease in diet-induced obesity mice by enhancing hepatic insulin and AMPK signaling independently of FGF21 pathway. Metabolism 2013;62:1239-49.

7. Zhong H, Chen K, Feng M, et al. Genipin alleviates high-fat diet-induced hyperlipidemia and hepatic lipid accumulation in mice via miR-142a-5p/SREBP-1c axis. FEBS J 2018;285:501-17.

8. Guan L, Gong D, Yang S, et al. Genipin ameliorates dietinduced obesity via promoting lipid mobilization and browning of white adipose tissue in rats. Phytother Res 2018;32:723-32.

9. Regulska-Ilow B, Biernat J, Grajeta H, et al. Influence of bioflavonoids from the radix extract of Scutellaria baicalensis on the level of serum lipids, and the development of laboratory rats fed with fresh and oxidized fats. Nahrung 2004;48:123-8.

10. Guo HX, Liu DH, Ma Y, et al. Long-term baicalin administration ameliorates metabolic disorders and hepatic steatosis in rats given a high-fat diet. Acta Pharmacol Sin
Yao et al. Yin Zhi Huang ameliorates obesity and hapatic steatosis

2009;30:1505-12.

11. Chen SP, Tian LL, Liu FL. Clinical observation of Yinzhihuang Oral Liquid on prevention of the premature infantile jaundice. Chin J Integr Med 2009;15:299-302.

12. Yang R, Fang L, Li J, et al. Separation of Five Iridoid Glycosides from Lonicerae Japonicae Flos Using HighSpeed Counter-Current Chromatography and Their AntiInflammatory and Antibacterial Activities. Molecules 2019. doi: 10.3390/molecules24010197.

13. Gao Y, Tang H, Xiong L, et al. Protective Effects of Aqueous Extracts of Flos lonicerae Japonicae against Hydroquinone-Induced Toxicity in Hepatic L02 Cells. Oxid Med Cell Longev 2018;2018:4528581.

14. Ameer F, Scandiuzzi L, Hasnain S, et al. De novo lipogenesis in health and disease. Metabolism 2014;63:895-902.

15. Donnelly KL, Smith CI, Schwarzenberg SJ, et al. Sources of fatty acids stored in liver and secreted via lipoproteins in patients with nonalcoholic fatty liver disease. J Clin Invest 2005;115:1343-51.

16. Ipsen DH, Lykkesfeldt J, Tveden-Nyborg P. Molecular mechanisms of hepatic lipid accumulation in non-alcoholic fatty liver disease. Cell Mol Life Sci 2018;75:3313-27.

17. Zhang L, Keung W, Samokhvalov V, et al. Role of fatty acid uptake and fatty acid beta-oxidation in mediating insulin resistance in heart and skeletal muscle. Biochim Biophys Acta 2010;1801:1-22.

18. Schreurs M, Kuipers F, van der Leij FR. Regulatory enzymes of mitochondrial beta-oxidation as targets for treatment of the metabolic syndrome. Obes Rev 2010;11:380-8.

19. Li Y, Xu S, Mihaylova MM, et al. AMPK phosphorylates and inhibits SREBP activity to attenuate hepatic steatosis and atherosclerosis in diet-induced insulin-resistant mice. Cell Metab 2011;13:376-88.

20. Park SH, Gammon SR, Knippers JD, et al. Phosphorylation-activity relationships of AMPK and acetyl-CoA carboxylase in muscle. J Appl Physiol (1985) 2002;92:2475-82.

21. Zou Y, Li S, Li Z, et al. MiR-146a attenuates liver fibrosis by inhibiting transforming growth factor-beta1 mediated epithelial-mesenchymal transition in hepatocytes. Cell Signal 2019;58:1-8.

22. Liu XJ, Duan NN, Liu C, et al. Characterization of a murine nonalcoholic steatohepatitis model induced by high fat high calorie diet plus fructose and glucose in drinking water. Lab Invest 2018;98:1184-99.

23. Zeng J, Wang SJ, Li YM, et al. Yinzhihuang oral liquid in 
the treatment of neonatal jaundice: a meta-analysis. Pharm Biol 2017;55:554-9.

24. Hong JH, Hwang EY, Kim HJ, et al. Artemisia capillaris inhibits lipid accumulation in 3T3-L1 adipocytes and obesity in C57BL/6J mice fed a high fat diet. J Med Food 2009;12:736-45.

25. Jung HA, Park JJ, Islam MN, et al. Inhibitory activity of coumarins from Artemisia capillaris against advanced glycation endproduct formation. Arch Pharm Res 2012;35:1021-35.

26. Kojima K, Shimada T, Nagareda Y, et al. Preventive effect of geniposide on metabolic disease status in spontaneously obese type 2 diabetic mice and free fatty acid-treated HepG2 cells. Biol Pharm Bull 2011;34:1613-8.

27. Sheng L, Qian Z, Zheng S, et al. Mechanism of hypolipidemic effect of crocin in rats: crocin inhibits pancreatic lipase. Eur J Pharmacol 2006;543:116-22.

28. Li Y, Cai W, Weng X, et al. Lonicerae Japonicae Flos and Lonicerae Flos: A Systematic Pharmacology Review. Evid Based Complement Alternat Med 2015;2015:905063.

29. Bochoráková H, Paulova H, Slanina J, et al. Main flavonoids in the root of Scutellaria baicalensis cultivated in Europe and their comparative antiradical properties. Phytother Res 2003;17:640-4.

30. Li HB, Jiang Y, Chen F. Separation methods used for Scutellaria baicalensis active components. J Chromatogr B Analyt Technol Biomed Life Sci 2004;812:277-90.

31. Xi Y, Wu M, Li H, et al. Baicalin Attenuates High Fat Diet-Induced Obesity and Liver Dysfunction: DoseResponse and Potential Role of CaMKKbeta/AMPK/ACC Pathway. Cell Physiol Biochem 2015;35:2349-59.

32. Dai J, Liang K, Zhao S, et al. Chemoproteomics reveals

Cite this article as: Yao Q, Li S, Cheng X, Zou Y, Shen Y, Zhang S. Yin Zhi Huang, a traditional Chinese herbal formula, ameliorates diet-induced obesity and hepatic steatosis by activating the AMPK/SREBP-1 and the AMPK/ACC/CPT1A pathways. Ann Transl Med 2020;8(5):231. doi: 10.21037/ atm.2020.01.31 baicalin activates hepatic CPT1 to ameliorate diet-induced obesity and hepatic steatosis. Proc Natl Acad Sci U S A 2018;115:E5896-E5905.

33. Kohjima M, Higuchi N, Kato M, et al. SREBP-1c, regulated by the insulin and AMPK signaling pathways, plays a role in nonalcoholic fatty liver disease. Int J Mol Med 2008;21:507-11.

34. Munday MR. Regulation of mammalian acetyl-CoA carboxylase. Biochem Soc Trans 2002;30:1059-64.

35. Liu Y, Beyer A, Aebersold R. On the Dependency of Cellular Protein Levels on mRNA Abundance. Cell 2016;165:535-50.

36. Pan L, Wang W, Shi F, et al. Exploratory Pharmacokinetics of Geniposide in Rat Model of Cerebral Ischemia Orally Administered with or without Baicalin and/or Berberine. Evid Based Complement Alternat Med 2013;2013:349531.

37. Huang W, Zhang J, Moore DD. A traditional herbal medicine enhances bilirubin clearance by activating the nuclear receptor CAR. J Clin Invest 2004;113:137-43.

38. Dong B, Saha PK, Huang W, et al. Activation of nuclear receptor CAR ameliorates diabetes and fatty liver disease. Proc Natl Acad Sci U S A 2009;106:18831-6.

39. Sberna AL, Assem M, Gautier T, et al. Constitutive androstane receptor activation stimulates faecal bile acid excretion and reverse cholesterol transport in mice. J Hepatol 2011;55:154-61.

40. Xu B, Li YL, Xu M, et al. Geniposide ameliorates TNBS-induced experimental colitis in rats via reducing inflammatory cytokine release and restoring impaired intestinal barrier function. Acta Pharmacol Sin 2017;38:688-98. 


\section{Supplementary}

\section{Cell viability assays}

The immortalized human hepatocyte cell line L02 was a gift from liver cancer center of Zhongshan Hospital and cultured in Dulbecco's modified Eagle's medium (HyClone Laboratories, Logan, UT, USA) supplemented with $10 \%$ fetal bovine serum (Gibco, Carlsbad, CA, USA), 1\% penicillin and streptomycin. Cell viability was assessed by the Cell Counting Kit-8-based spectrophotometric methods (Beyotime Institute Biotechnology, China) according to the manufacturer's protocol. Cells were seeded in 96-well microtiter plates at a density of $5 \times 10^{3}$ cells/well. After 6 hours, the culture medium was replaced with serum-free medium containing $\mathrm{YZH}$ with various volume concentrations $(0,1 \%, 1 \%, 10 \%, 20 \%)$ for 0,24 or $48 \mathrm{~h}$ at $37{ }^{\circ} \mathrm{C}$ and $5 \% \mathrm{CO}_{2}$. Following treatment, $10 \mu \mathrm{L}$ CCK8 solution was added in each cell and incubated for another 2 hours. Relative cytotoxicity was measured with EPOCH2 microplate reader (BioTek Instruments Inc., USA) at $450 \mathrm{~nm}$ absorbance. Cell viability was defined as relative to the vehicle-treated control, and the experiment was conducted three times independently.

Table S1 PCR primers used in the present study

\begin{tabular}{|c|c|c|}
\hline Genbank accession & Name & Sequence $\left(5^{\prime}-3^{\prime}\right)$ \\
\hline NM_007393.3 & & GTAACAGTCCGCCTAGAAGCAC \\
\hline \multirow[t]{2}{*}{ NM_001313979.1 } & SREBP-1 & TGACCCGGCTATTCCGTGA \\
\hline & & CTGGGCTGAGCAATACAGTTC \\
\hline NM_007988.3 & & TGGGTAATCCATAGAGCCCAG \\
\hline \multirow[t]{2}{*}{ NM_013495 } & CPT1A & CCAGGCTACAGTGGGACATT \\
\hline & & GAACTTGCCCATGTCCTTGT \\
\hline NM_008255.2 & Hmgcr & AGCTTGCCCGAATTGTATGTG \\
\hline NM_017399.5 & & CTGACACCCCCTTGATGTCC \\
\hline \multirow[t]{2}{*}{ NM_007381.4 } & Lcad & TCTTTTCCTCGGAGCATGACA \\
\hline & & GACCTCTCTACTCАСTTCTCCAG \\
\hline \multirow[t]{2}{*}{ NM_008509.2 } & LPL & GGGAGTTTGGCTCCAGAGTTT \\
\hline & & TGTGTCTTCAGGGGTCCTTAG \\
\hline \multirow[t]{2}{*}{ NM_007824.3 } & CYP7a1 & GGGATTGCTGTGGTAGTGAGC \\
\hline & & GGTATGGAATCAACCCGTTGTC \\
\hline NM_021282.3 & CYP2E1 & GGTATGGAATCAACCCGTTGTC \\
\hline
\end{tabular}


Table S2 Effects of different dose of YZH on the body weight, organs mass, plasma parameters in the animal experiment

\begin{tabular}{|c|c|c|c|c|}
\hline Parameter & Chow & HFD & \multicolumn{2}{|c|}{ YZH } \\
\hline \multicolumn{5}{|c|}{ Body weight measurements } \\
\hline Initial body weight (g) & $21.87 \pm 0.61$ & $21.45 \pm 0.72$ & $20.79 \pm 1.05$ & $20.66 \pm 0.78$ \\
\hline Final body weight (g) & $32.24 \pm 0.60$ & $44.34 \pm 2.21^{\star \star \star}$ & $39.64 \pm 1.10^{\# \# \#}$ & $34.10 \pm 0.97^{\# \# \#}$ \\
\hline Epididymal fat pad (g) & $0.33 \pm 0.08$ & $1.97 \pm 0.25^{\star \star \star}$ & $1.44 \pm 0.21^{\# \#}$ & $0.86 \pm 0.19^{\# \# \#}$ \\
\hline Inguinal fat pad (g) & $0.31 \pm 0.16$ & $1.45 \pm 0.44^{\star \star \star}$ & $0.81 \pm 0.28^{\#}$ & $0.53 \pm 0.20^{\# \#}$ \\
\hline Liver weight (g) & $1.12 \pm 0.05$ & $1.54 \pm 0.12^{\star \star \star}$ & $1.29 \pm 0.11^{\# \# \#}$ & $1.17 \pm 0.07^{\# \#}$ \\
\hline \multicolumn{5}{|c|}{ Serum biochemical variables } \\
\hline ALT & $31.21 \pm 2.24$ & $62.79 \pm 6.83^{\star \star \star}$ & $48.10 \pm 5.17^{\# \#}$ & $34.10 \pm 2.85^{\# \# \#}$ \\
\hline Creatinine & $68.06 \pm 13.23$ & $68.54 \pm 8.36$ & $67.86 \pm 12.41$ & $70.48 \pm 13.74$ \\
\hline Urea nitrogen & $7.94 \pm 0.85$ & $7.44 \pm 0.54$ & $7.31 \pm 0.94$ & $7.40 \pm 0.71$ \\
\hline Amylase & $508.93 \pm 41.94$ & $530.53 \pm 24.68$ & $533.92 \pm 47.96$ & $490.23 \pm 57.09$ \\
\hline
\end{tabular}

${ }^{*} \mathrm{P}<0.05$, ${ }^{*} \mathrm{P}<0.01$, ${ }^{* \star *} \mathrm{P}<0.001$ compared with the Chow group according to unpaired two-tailed Student's $t$-test; ${ }^{\#} \mathrm{P}<0.05$, ${ }^{\# \#} \mathrm{P}<0.01$, ${ }^{\# \# \#} \mathrm{P}<0.001$ compared with the HFD group according to unpaired two-tailed Student's $t$-test. YZH, Yin Zhi Huang; HFD, high-fat diet; TC, total cholesterol; TG, triglyceride; AST, aspartate transaminase; ALT, alanine transaminase.

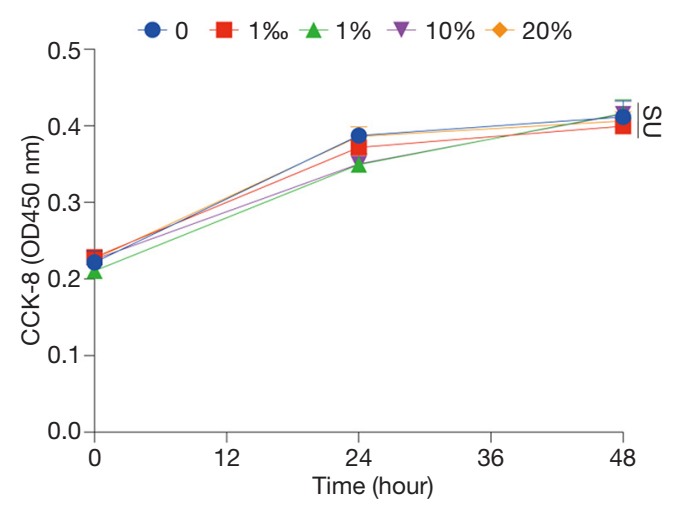

Figure S1 Cell viabilities of L02 cell line incubated with YZH at volume concentration from 1\%o to $20 \%$ for 0 , 24 or 48 h. ns means no statistical difference. YZH, Yin Zhi Huang. 

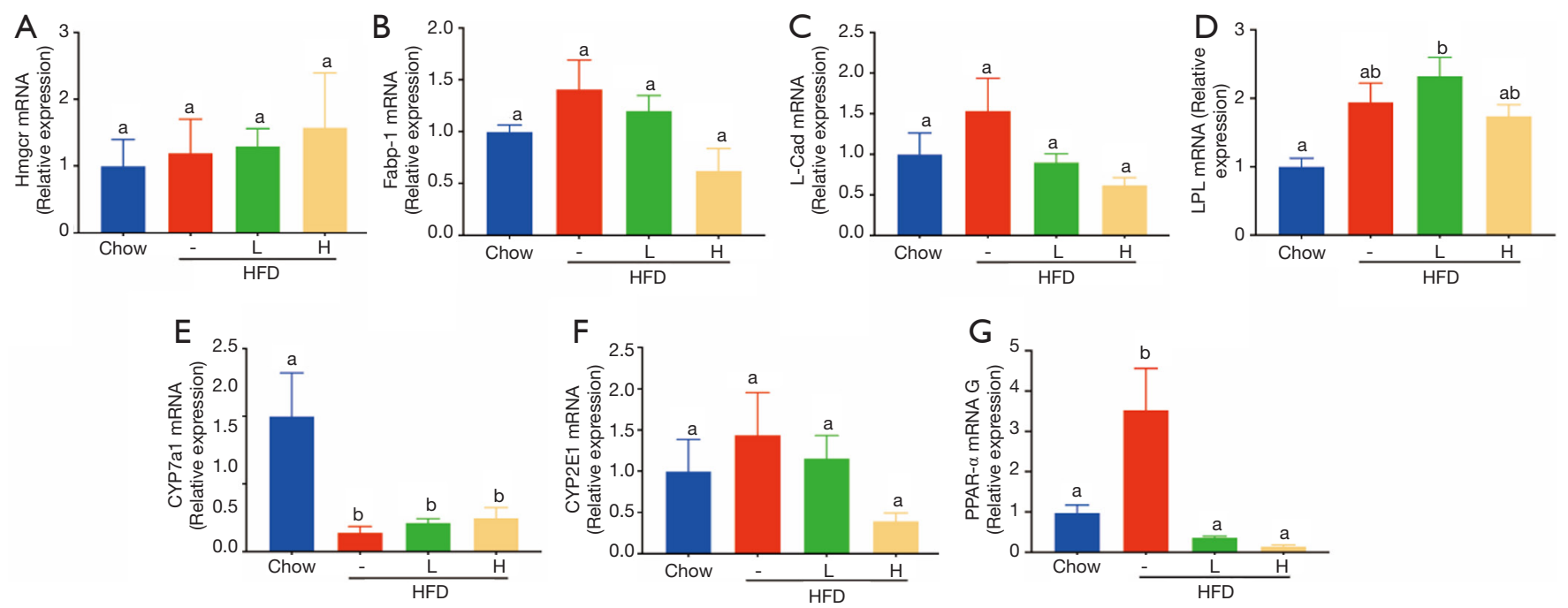

Figure S2 mRNA levels of genes related to fatty acid synthesis, $\beta$-oxidation, bile acid and cholesterol metabolism in liver. (A) 3-hydroxy3-methylglutaryl CoA reductase (Hmgcr), (B) fatty acid binding protein 1 (Fabp1), (C) long chain acyl-CoA dehydrogenase (Lcad), (D) lipoprotein lipase (Lpl), (E) Cholesterol 7a-hydroxylase (Cyp7a1), (F) Cytochrome P450 Family 2 Subfamily E Member 1(CYP2E1), and (G) peroxisome proliferation-activated receptor (PPAR)- $\alpha$ levels were analysed by qRT-PCR. The values were presented as mean \pm S.E.M. $(\mathrm{n}=6$ per group). Graph bars with different letters on top represent statistically significant results $(\mathrm{P}<0.05)$ based on Newman-Keuls post hoc oneway ANOVA analysis, whereas bars with the same letter correspond to results that show no statistically significant differences. 